\title{
DESIGN AND ANALYSIS OF DISCONTINUOUS MODE SERIES RESONANT CONVERTER
}

\author{
Wing C. Ho M. H. Pong \\ Department of Electrical and Electronic Engineering \\ The University of Hong Kong
}

Abstract - This paper aims at designing a low power, off-line, high efficiency, complex in size, twin-capacitors half-bridge series resonant converter. It gives steps by steps design procedures of the discontinuous mode series resonant converter starting from power stage circuit design to feedback loop control design. The small signal analysis is based on the injected-absorbed current method.

\section{INTRODUCTION}

In the design process of a low power, off-line, high efficiency converter, the size and component counts are the critical factors which are concerned by many designers. Twin-capacitors half-bridge series resonant converter [1] provides a way in which bulky components can be significantly reduced. This paper aims at the design and analysis of the twin-capacitors half-bridge resonant converter running in the discontinuous mode of operation. Steps by steps design procedures for the power stage and the small signal analysis, which is based on the injected-absorbed current method [2,3], for the loop control are given. Computer simulation and power circuit prototype have been used to verify the process. The captured waveforms will also be discussed.

\section{POWER STAGE}

Comparing with the traditional half-bridge series resonant converter, Fig. 1, the twin-capacitors half-bridge series resonant converter, Fig. 2, uses two very small size resonant capacitors, $\mathrm{Co}$, at the positions of the input bulk capacitors, Cin. Actually the Thevenin equivalent circuits at position $\mathrm{X}$ for both Fig. 1 and Fig. 2 are the same, provided that Cin is much greater than $\mathrm{Co}$. And the equivalent resonant capacitor, $\mathrm{Cr}$, is equal to $2 \mathrm{Co}$.

There are three modes of operation in the full-wave discontinuous mode:

(1) time interval $0<t<$ To; see Fig. 3

where $\mathrm{To}_{\mathrm{O}}=$ half of resonant period

Taking Laplace transform,

$$
\frac{(e-\mu)}{s}-V_{C r}(s)-s L_{r} I_{L r}(s)=0
$$

where $I_{L V}(0)=0$

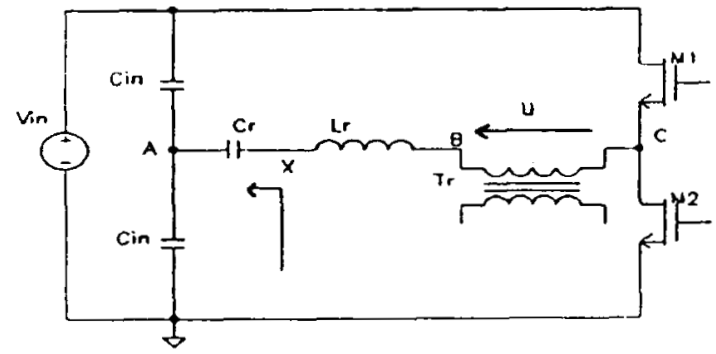

Fig. 1 Traditional half-bridge SCR

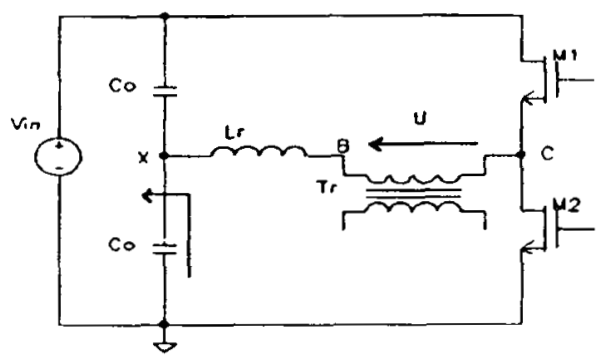

Fig. 2 Twin-capacitors half-bridge SCR

$I_{L r}(s)-s C_{r} V_{C r}(s)=0$

where $V_{C_{r}}(0)=-V_{m}$

(2) time interval To $<\mathrm{t}<2$ To; see Fig. 4

$\frac{(e-u)}{s}-V_{C r}(s)-s L_{r} I_{L r}(s)=0$

where $I_{L r}(T o)=0$

$I_{L r}(s)-s C_{r} V_{C r}(s)=0$

where $V_{C r}(T o)=V_{a}$

(3) time interval 2 To $<\mathrm{t}<\mathrm{Ts}$; see Fig. 5

where $T s=$ half of the switching period

$i_{L, 3}=0$

$V_{C r 3}=V_{m}$

Solving for equation (1) to (6) and taking the inverse Laplace transform, one can find that:

(1) $i_{L r 1}=\frac{\left(e-u-V_{m}\right)}{z_{o}} \sin \omega_{o} t$

where $e=\frac{V_{\text {in }}}{2} ; \mathrm{u}=$ reflected output voltage

$I_{p k \mathrm{l}}=\frac{e}{Z_{0}}(1+M)$

i.e. peak current at $\omega_{o} t=\pi / 2$

$V_{C r 1}=(e-u)-\left(e-u-V_{m}\right) \cos \omega_{o} t$

$V_{p k 1}=2 e$

i.e. peak voltage at $\omega_{o} t=\pi$ 


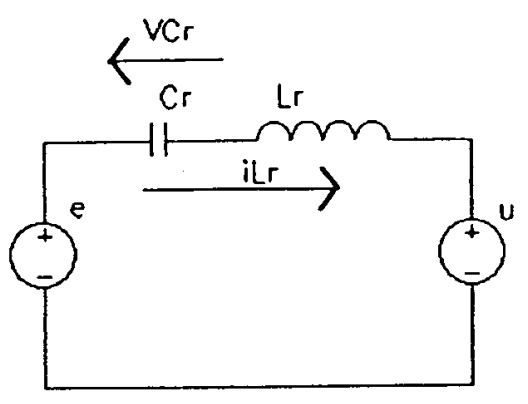

(a) time : $0<\mathbf{t}<$ To

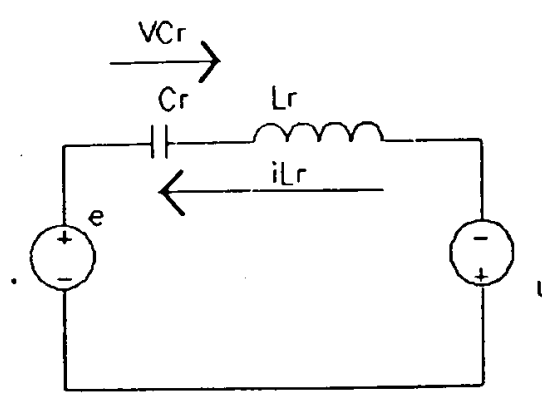

(b) time : To $<\mathrm{t}<2$ To

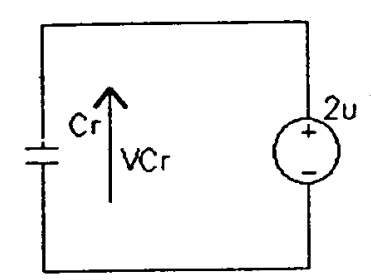

(c) time : 2 To $<\mathrm{t}<\mathrm{Ts}$

Fig. 3 Equivalent circuits of discontinuous mode series resonant converter

(2) time interval To $<\mathrm{t}<2$ To;

$i_{L n 2}=\frac{\left(e+u-V_{a}\right)}{Z o} \sin \omega_{o}(t-T o)$

$I_{p k 2}=-\frac{e(1-M)}{Z o}$

i.e. peak current at $\omega_{o} t=3 \pi / 2$

$V_{C r 2}=(e+u)-\left(e+u-V_{a}\right) \cos \omega_{o}(t-T o)$

$V_{p k 2}=2 u$ i.e. peak voltage at $\omega_{o} t=2 \pi$

(3) time interval 2 To $<\mathrm{t}<\mathrm{Ts}$

$i_{L r 3}=0$

$V_{C r 3}=2 u$

Fig. 4 shows the computer simulated waveforms.
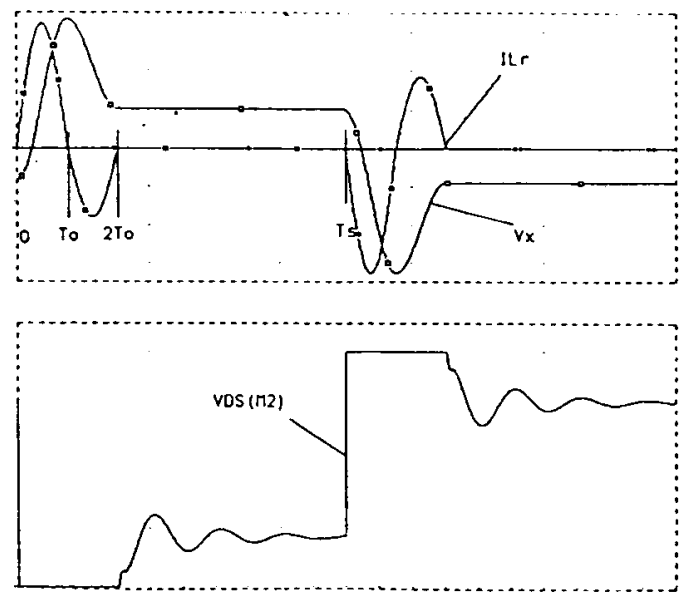

Fig. 4 Simulated waveforms of half-bridge series resonant converter

\section{DC CONVERSION RATIO AND AVERAGE OUTPUT CURRENT}

By taking the average resonant inductor current over half switching period, it can be found that:

$$
\begin{aligned}
i_{o} & =\frac{2}{T_{s}}\left[\int_{0}^{T o} i_{L r 1} d t-\int_{T o}^{2 T o} i_{L r 2} d t\right] \\
\Rightarrow i_{o} & =\frac{2 e f n}{\pi Z o} \quad \text { where fn=fs/fo }
\end{aligned}
$$

And substituting Io $=u /$ Ro into equation (18), one gets

$$
M=\frac{u}{e}=\frac{2 f n r}{\pi} \quad \text { where } \mathrm{r}=\mathrm{Ro} / \mathrm{Zo}
$$

Fig. 5 shows the plot of $M$ as a function of $f($ with different values of $r$. One can put the max. (or min.) value of line (or load) condition on the plot for design purpose, with fs less than 0.5 fo.

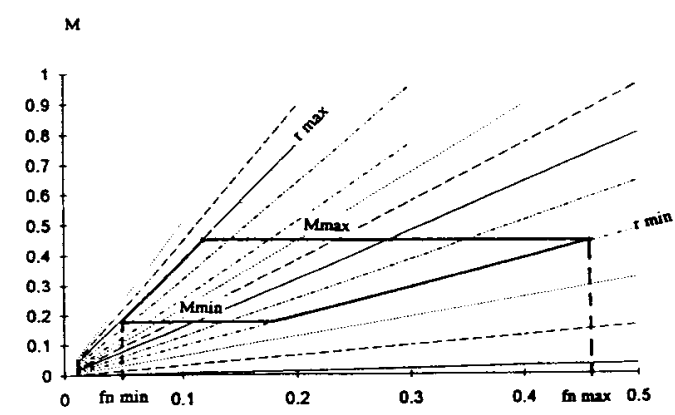

Fig. 5 Plot of voltage conversion ratio of discontinuous mode full-wave half-bridge SCR

From equation (17), by substituting $V m=-2 u$, it is found that

$$
V_{a}=\frac{u}{4 / s C r R_{0}}
$$

Using the same approach of finding Io, one can find the average input current as

$$
I_{\text {in }}=\frac{2}{T s}\left[\int_{0}^{T o} i_{L r 1} d t+\int_{T o}^{2 T o} i_{L r 2} d t\right]
$$

By substituting $\mathrm{Va}=2 \mathrm{e}$, the value of $\mathrm{Vm}$ is

$$
V_{m}=-\frac{u^{2}}{4 e f s r_{o}}
$$

The values of $\mathrm{Vm}$ and $\mathrm{Va}$ are used for determining the small signal in later section. 


\section{SMALL SIGNAL ANALYSIS}

The output current, io, that is derived from equation (17), is being injected into the output loading, Fig. 6.

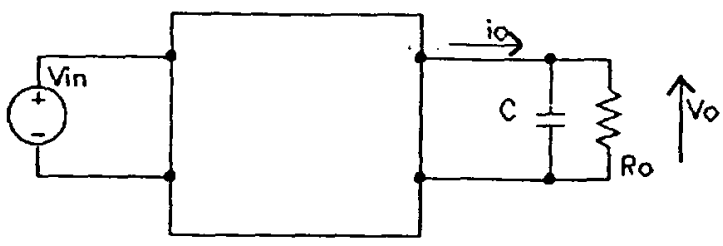

Fig; 6 Average output current is being injected into output loading

One can rewrite equation (17) as follows:

$$
\begin{aligned}
i_{o}= & 2 f s\left\{\frac { 1 } { Z o \omega _ { 0 } } \left[(e-u-V m)\left(1-\cos \omega_{o} t a\right)\right.\right. \\
& \left.-(e+u-V a)\left(1-\cos \left(2 \pi-\omega_{o} t a\right)\right]\right\}
\end{aligned}
$$

where $t a=\frac{1}{\omega_{o}} \cos ^{-1}\left(\frac{e-\mu-V a}{e-u-V_{m}}\right)$

The total differentials of the output current [2,3], equation (23), can be expressed in terms of input (e), output (u) and control signal (fs) as

$$
d i_{o}=\frac{\partial i_{o}}{\partial s} d f_{s}+\frac{\partial i_{o}}{\partial u} d u+\frac{\partial i_{o}}{\partial e} d e
$$

Taking the Laplace transform of (25), one gets

$$
\begin{aligned}
& I_{o}(s)=A F_{s}(s)-B V_{o}(s)+C E(s) \\
& \text { where } A=\frac{\partial i_{o}}{\partial f_{s}}, B=-\frac{\partial i_{o}}{\partial u}, C=\frac{\partial i_{o}}{\partial e}
\end{aligned}
$$

The numerical values of $A, B$ and $C$ can be obtained by substituting the dc values of $e, u, f s, V m$ and Vs. Now changing (26) into the form of

$$
E_{\text {out }}(s)=\frac{A}{B} F_{s}(s)+\frac{C}{B} E(s)=I_{o}(s) Z_{\text {out }}+V_{o}(s)
$$

$$
\text { where } Z_{\text {out }}=\frac{1}{B}
$$

Fig. 7 shows the equivalent circuit of equation (27).

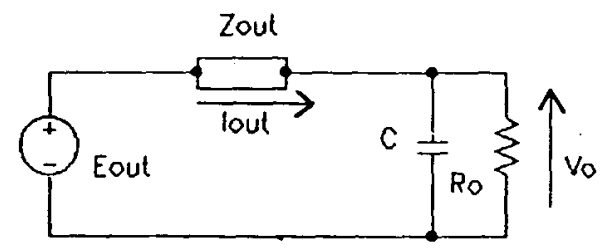

Fig. 7 Modified output circuit diagram From Fig. 8, one can express the Vo(s) as

$V_{o}(s)=E_{\text {out }}(s) \frac{Z o}{Z_{\text {out }}+Z o}$

where $Z o=\frac{R_{0}}{1+s C R_{0}}$

$\Rightarrow V_{o}(s)=H_{e} F_{s}(s)+G E(s)$

where $H e=\frac{A R_{0}}{1+B R_{o}+s C R_{0}}$

$$
G=\frac{C R_{0}}{1+B R_{o}+S C R_{o}}
$$

The open loop output-to-control transfer function can then be directly obtained by letting $\mathrm{E}(\mathrm{s})$ equal to zero. It is cleared that the transfer function is in first order.

\section{DESIGN EXAMPLE}

An off-line twin-capacitors half-bridge series resonant converter has been built. The specification of the converter is:

$\mathrm{Vin}=130 \mathrm{Vdc}$

$\mathrm{Vo}_{0}=5 \mathrm{~V}$

$\mathrm{Ro}=2.5 \mathrm{ohm}$

resonant freq. $=700 \mathrm{KHz}$

operating freq. $=130 \mathrm{KHz}$

resonant cap. $=12.8 \mathrm{nF}$

resonant inductor $=8 \mathrm{uH}$

output filter cap. $=47 \mathrm{uF}$

gain of $\mathrm{VCO}=-100 \mathrm{KHz} / \mathrm{V}$

The measured waveforms are shown in Fig. 8.

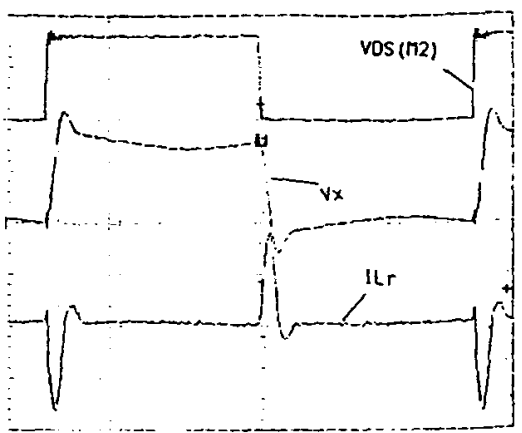

Fig. 8 Waveforms measured from a prototype:

(a) drain-source voltage of M2, (b) voltage at point $X$, (c) resonant inductor current.

Fig. 9 shows the simulated Bode plot of the open loop output-to-control transfer function. Fig; 10 and Fig. 11 show the block diagram of vco and converter, and the simulated and measured Bode plot of the open loop output-to-vco transfer function, respectively.
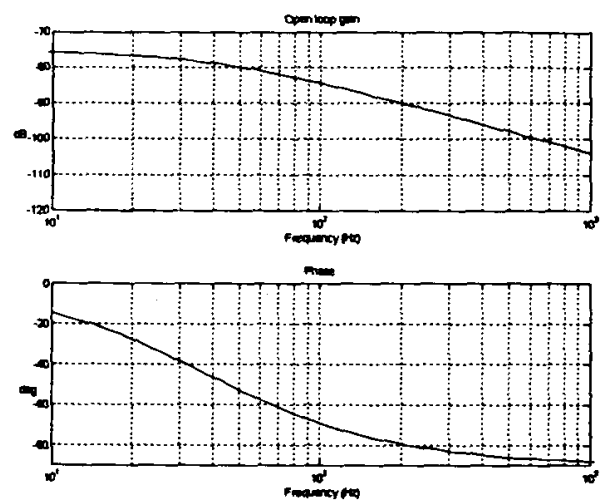

Fig. 9 Simulated Bode plot of the transfer function $\mathrm{He}$ 


\section{REFERENCES}

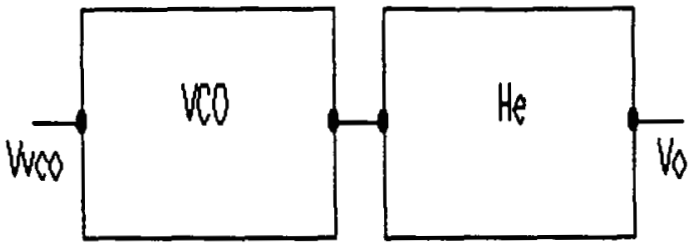

Fig. 10 Block diagram of VCO and converter
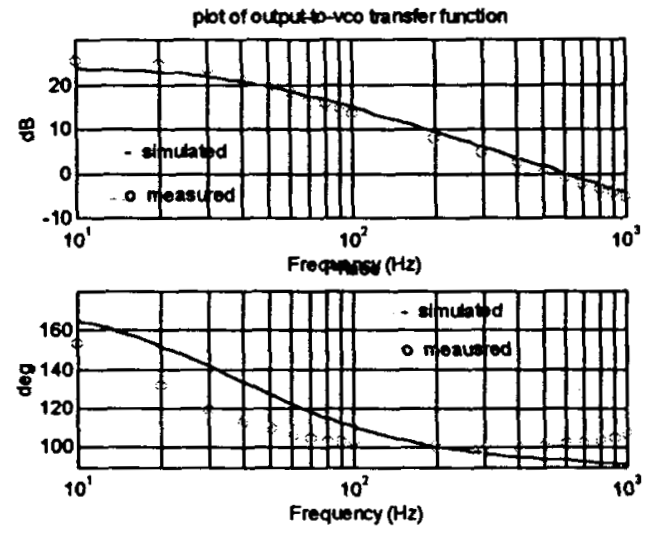

Fig. 11 Simulated and measured gain-phase of the open loop output-to-VCO transfer function

It is observed that the gain plot reveals a good result of prediction. The phase plot shows a similar trend of the phase shift. The deviation of the simulated and measured phase shift may be due to the unpredictable phase shift from the VCO.

\section{CONCLUSION}

The power stage of the discontinuous mode series resonant is easy to analyse. The DC conversion ratio is a linear function of normalized frequency. The small signal transfer function of the converter is in first order. Although the injected-absorbed current method is not an exact method to analyse the small signal of the converter, it gives an enough accurate result, and fast and simple way to handle the complexity of the dynamic analysis.
1. R. King, T.A. Stuart, "A Normalized Model for the Half-Bridge Series Resonant Converter", IEEE Trans. on Aerospace and Electronic Systems; Vol. $A S-17$, No. 3; May 1981; pp 181- 190.

2. A.S. Kislovski, "A Contribution to Steady-State Modeling of Half-Bridge Series Resonant Power Cells", IEEE Trans. on Power Electronics; Vol. PE-1, No. 3; July 1986; $p p$ 161 - 166.

3. A.S. Kislovski, R. Redl, N.O. Sokal, "Dynamic Analysis of Switching-Mode $D C / D C$ Converters", Van Nostrand Reinhold, 1991. 